\title{
X-RAY SPECTRAL PROPERTIES OF RADIO GALAXIES
}

\author{
H. INOUE \\ Institute of Space and Astronautical Science \\ 3-1-1 Yoshinodai, Sagamihara, Kanagawa 229, Japan
}

$\mathrm{X}$-ray spectral properties of radio galaxies are reviewed in comparison with those of Seyfert galaxies, mainly based on results from Japanese X-ray astronomy satellites, Ginga and ASCA.

\section{Spectral Indices in $2-10 \mathrm{keV}$}

Figure 1 shows energy-spectral indices in $2-10 \mathrm{keV}$ as a function of the cosmological redshift of various kinds of active galactic nuclei (AGN) obtained with Ginga and ASCA. As seen in this figure, all kinds of AGN including radio galaxies have the spectral indices in a fairly narrow range in 0.5 - 1.0 except some BL Lac objects, although radio-quiet quasars seem to have slightly steeper spectra than radio-loud quasars (Williams et al. 1992). Steeper spectra of some BL Lac objects are considered to be due to a large blue shift of a high energy end of a synchrotron spectrum to the $\mathrm{X}$-ray band (e.g. Macomb et al. 1995).

\section{Ratio of X-Ray Flux to Far Infrared Flux}

\subsection{SEYFERT GALAXIES}

In the unified model for Seyfert galaxies, a central power house is considered to be partially surrounded by a molecular/dust torus and whether a molecular torus is in or out of the line of sight to a central nucleus determines whether the source is identified to be a Seyfert 2 or 1 galaxy, respectively. Since a part of X-rays should be absorbed and be reemitted in far-infraredrays by the torus, a far-infrared emission should be observed irrespectively of whether a source is a Seyfert 1 or 2 . In fact, far-infrared emissions are generally detected from Seyfert galaxies.

Although no obscuration of optical and X-ray emission from the nucleus is observed from Seyfert 1 galaxies, their far-infrared fluxes are generally 


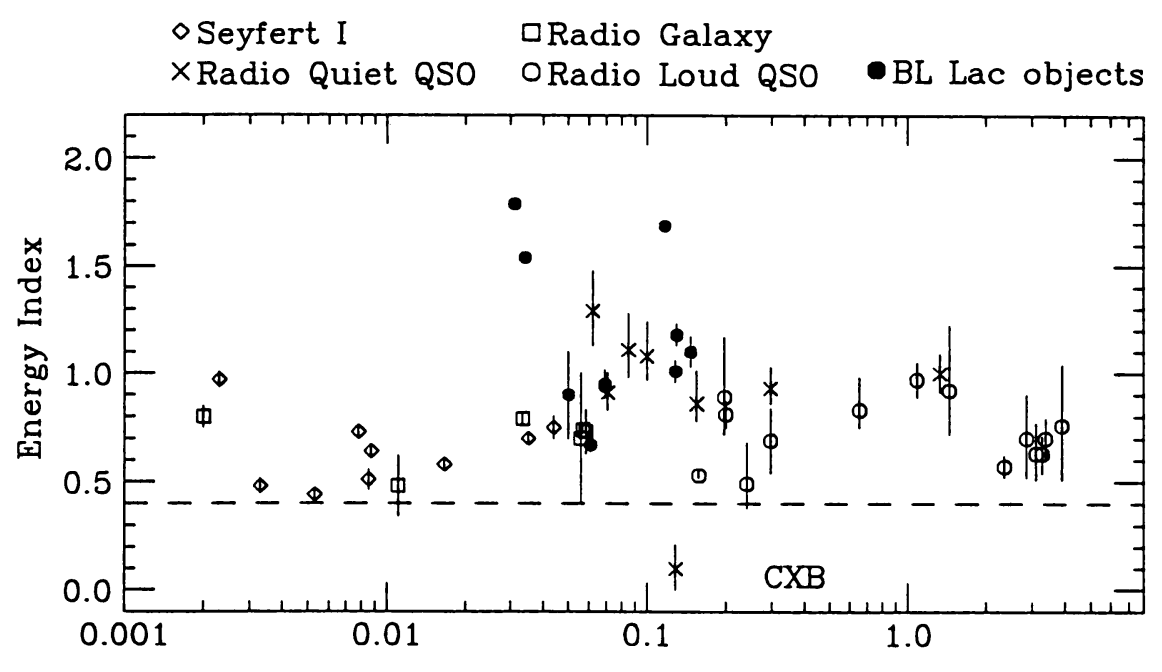

Redshift Z

Figure 1. Energy indices in $2-10 \mathrm{keV}$ as a function of the cosmological redshift, $\mathrm{z}$, of various kinds of AGNs obtained with Ginga and ASCA.

comparable to the $\mathrm{X}$-ray fluxes. If we calculate the ratio of the $\mathrm{X}$-ray flux $(2-10 \mathrm{keV})$ to the far-infrared flux (60-100 $\mu \mathrm{m}$ flux defined in the IRAS galaxy catalogue, Ver. 2, 1989) for 20 samples observed with Ginga (Nandra and Pounds 1994), we see them distribute in a range from 0.1 to 1 with a mean of 0.5. This is consistent with a conjecture that the far-infrared emissions are from dust tori heated by $\mathrm{X}$-rays from nuclei.

The X-ray to far-IR flux ratio tends to be smaller in Seyfert 2 galaxies than those of Seyfert 1 galaxies. Most of Seyfert 2 galaxies observed with Ginga (Awaki 1992 and references therein) show heavy absorption in the $\mathrm{X}$-ray band and it is consistent with their absence of optical broad lines probably due to obscuration of central broad line regions. The ratios of the observed X-ray flux to the far-IR flux of these sources are in a range $10^{-2}-10^{-1}$. If we calculate the intrinsic $X$-ray flux by correcting the fluxdecrease by absorption, their ratios of the intrinsic X-ray flux to the far-IR flux become consistent with those of Seyfert 1 galaxies.

Some of the Seyfert 2 galaxies, however, show no large absorption but have ratios of $\mathrm{X}$-ray to far-IR flux as small as $10^{-4}-10^{-3}$. These exhibit a prominent $\mathrm{K}$-emission line of iron in low ionization states with equivalent width of about $1.5 \mathrm{keV}$. These iron lines are interpreted to be due to fluorescent process of $\mathrm{X}$-rays by some ambient matter to a central X-ray source and their equivalent width can be calculated on an assumption of a simple geometry (see e.g. Inoue 1985). Then, such a high equivalent width 
of iron fluorescent line as $\sim 1.5 \mathrm{keV}$ can be obtained only when the central $\mathrm{X}$-ray source is completely obscured by some matter and $\mathrm{X}$-rays scattered by the ambient matter is only observed as continuum. This well explains the very low X-ray to far-IR flux ratios of these sources. In fact, Ginga found presence of heavily absorbed component in the energy range above $10 \mathrm{keV}$ from one of such Seyfert 2 galaxies showing a prominent iron emission line, NGC4945 (Iwasawa et al. 1993). The ratio of the intrinsic X-ray flux after correcting for absorption to the far-IR flux is well in the range of Seyfert 1 galaxies.

These are all consistent with the unified scheme of Seyfert galaxies.

\subsection{RADIO GALAXIES}

Ratio of X-ray flux to far-IR flux after correcting for absorption was calculated for five radio galaxies observed with Ginga and/or ASCA (3C120, 3C390.3, 3C445, Cen A and Cyg A) and again ranges around $0.1-1.0$. This is consistent with unification between radio galaxies and Seyfert galaxies.

\section{Breadth of iron emission line}

An ASCA observation of the Seyfert 1 galaxy, MCG-6-30-15, revealed a broad feature of the iron emission line (Tanaka et al. 1995: see the left panel of Fig.2). Most of the line flux is strongly redshifted from the rest energy and the large full width at zero intensity indicates that the line comes from matter moving with velocity of $\sim 100,000 \mathrm{~km} \mathrm{~s}^{-1}$ (a third of the light velocity). These properties are interpreted to be due to Doppler and general relativistic effect of matter circulating in an accretion disk very close to the central black hole (Tanaka et al. 1995; Fabian et al. 1995). Similar broad line-profiles have been detected from several Seyfert 1 galaxies (Otani 1995; Yaqoob et al. 1995; Iwasawa et al. 1995).

An ASCA observation of the broad line radio galaxy, 3C390.3 (Eracleous et al. 1995), however, didn't show such a broad line feature as in Seyfert 1 galaxy, as seen in Fig.2. The absence or weak appearance of the broad line feature in the radio galaxy might suggest lack of an inner line-reprocessing region in this source and be related to presence of the superluminal jet in this source (Alef et al. 1988).

\section{Summary}

Similarities and differences in X-ray spectral properties between radio galaxies and Seyfert galaxies have been studied. No large differences have been found in the spectral indices in $2-10 \mathrm{keV}$ and in the ratios of the X-ray flux to far-IR flux. The breadth of iron lines may be a difference between 

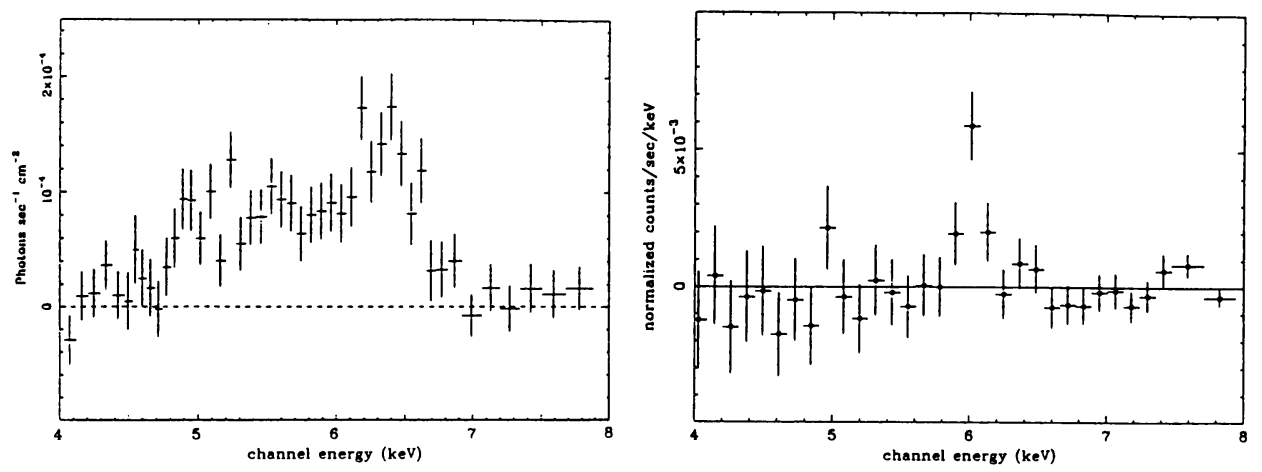

Figure 2. The line profile of iron $K_{\alpha}$ in the X-ray emission from MCG-6-30-15 ( $z=0.008$ : left panel) and from 3C390.3 ( $z=0.056$ : right panel).

them. Several Seyfert galaxies reveal a very broad feature of the iron emission line and the line properties are well interpreted by introducing Doppler and general relativistic effects on the line coming from a region very close to the central black hole. Whereas, the iron emission line from the broad line radio galaxy, 3C390.3 doesn't have such a broad feature. This may relate to presence of jets in radio galaxies, although further X-ray observations of radio galaxies are obviously necessary.

The author thanks H.Kubo, M.Sugizaki, T.Takahashi and A.Yamashita for their help in the preparation of this paper.

\section{References}

Alef, W. et al. (1988), A.Ap., 192, 53.

Awaki, H. (1992), in "Frontiers of X-ray Astronomy" ed. Y.Tanaka and K.Koyama, Universal Academy Press, Tokyo, p.537.

Eracleous, M., Halpern, J.P. and Livio, M. (1995), Ap.J., in press.

Fabian, F. et al. (1995), MNRAS, in press.

Inoue, H. (1985), Space Sci. Rev., 40, 317.

Iwasawa, K. et al. (1993), Ap.J., 408, 155.

Iwasawa, K. et al. (1995), MNRAS, in press.

Macomb, D.J. et al. (1995), Ap.J., 449, L99.

Nandra, K. and Pounds, K.A. (1994), MNRAS, 268, 405.

Otani, C. (1995), Ph.D.Thesis, Univ. of Tokyo.

Tanaka, Y. et al. (1995), Nature, 375, 659.

Williams, O.R. et al. (1992), Ap.J., 389, 157.

Yaqoob, T. et al. (1995), Ap.J., in press. 